\title{
Thermochromatography of Heavy Actinides - Determination of the Sublimation Enthalpy of Es*
}

\author{
By Steffen Taut ${ }^{1}$, S. Hübener', B. Eichler ${ }^{2}$, H. W. Gäggeler ${ }^{2,3}$, M. Schädel ${ }^{4}$ and I. Zvara ${ }^{5}$ \\ ${ }^{1}$ Forschungszentrum Rossendorf e.V., Institut für Radiochemie \\ 2 PSI Villigen, Labor für Radio- und Umweltchemie \\ ${ }^{3}$ Universität Bern, Departement für Chemie und Biochemie \\ 4 Gesellschaft für Schwerionenforschung Darmstadt \\ $s$ JINR Dubna, Flerov Laboratory of Nuclear Reactions
}

(Received November 18, 1996; accepted in revised form January 10, 1997)

Californium / Einsteinium / Fermium / Thermochromatography / Adsorption / Sublimation enthalpy

\begin{abstract}
Summary
The adsorption of Cf, Es, and Fm on several metals was studied thermochromatographically. In all systems investigated Es is adsorbed between the $\mathrm{Cf}$ and $\mathrm{Fm}$, due to the increasing metallic divalency in the second half of the actinide series. Based on the adsorption data and the experimentally proved correlation between adsorption and sublimation heats we calculated the Es sublimation enthalpy of $167 \mathrm{~kJ} / \mathrm{mol}$. This is significantly higher than the value obtained by direct vapour pressure measurements (133 $\mathrm{kJ} / \mathrm{mol}$ [1]). However, this value fits better to theory.
\end{abstract}

\section{Introduction}

The sublimation enthalpy, one of the fundamental metallic properties, is the room temperature analogue of the cohesive energy, which is connected directly with the valence electronic structure of the metal [2]. The sublimation enthalpy has been determined for the majority of the actinides by vapour pressure measurements; a compilation can be found in [3].

In addition to the disadvantage the handling the highly radioactive heavy actinides pose, these measurements with macroamounts of the metallic actinides were limited to those elements available in macroscopic quantities. However, the last three members of the actinide series can be produced only on heavy ion accelerators in non-weighable quantities.

As pointed out in [4], one method to obtain the sublimation enthalpies even for $\mathrm{Md}, \mathrm{No}$, and $\mathrm{Lr}$ is the thermochromatographic measurement of adsorption enthalpies of these metals which are in many cases correlated to the sublimation enthalpies. The use of concentrations much lower than necessary for monolayer coverage in this method has the advantage over methods using macroamounts in that simultaneous studies of different species are possible, since these species can not influence each other in their adsorption behaviour. This allows one to draw highly accurate

\footnotetext{
* Presented at the Fourth International Conference on Nuclear and Radiochemistry, Saint Malo, September 1996.
}

conclusions about the gradation of adsorption properties between species examined in one experiment. Furthermore, if there is a well known species among the substances under study, one has an inner standard, which gives the opportunity to proove the validity of the obtained absolute adsorption data.

The first step in this experimental approach is to measure an extensive amount of adsorption data of the actinides up to Fm as reference data for further experiments with the heaviest actinides.

Up to now, several thermochromatographic experiments have been carried out with Cf, Es and Fm on metal columns. In the first experiments, including even Md, Ca vapours were added to the chromatographic carrier gas in order to remove oxygen impurities [5, 6]. Another advantage was that this $\mathrm{Ca}$ was an internal standard for a divalent metal with a well-defined sublimation enthalpy. This added $\mathrm{Ca}$, however, had the serious drawback of making the interpretation of results difficult, because the macroamounts of $\mathrm{Ca}$ modified the chromatographic column. Further experiments showed that reliable results are possible without $\mathrm{Ca}$, if the apparatus is designed carefully [7]. Unfortunately, in these experiments the maximum temperature was not very high so that in many cases only lower limits of adsorption enthalpies could be obtained.

As the first part of a larger program, we had investigated the adsorption of Cf, Es and Fm with an apparatus, providing maximum temperatures up to $2100 \mathrm{~K}$, without the use of $\mathrm{Ca}$ vapours as reductant in the carrier gas. The aim was both to show the validity of the data obtained with our new thermochromatographic apparatus and to complete the series of adsorption data for Cf, Es and Fm from [7].

\section{Experimental}

\subsection{Thermochromatographic apparatus}

Fig. 1 shows the thermochromatographic setup. It is similar to that used in [7] but provides temperatures up to $2100 \mathrm{~K}$ in the hottest zone. The gradient oven is a commercial model LORA 36 from HTM Reetz $\mathrm{GmbH}$, Berlin. We made efforts to avoid seepage of oxygen into the chromatographic system: 


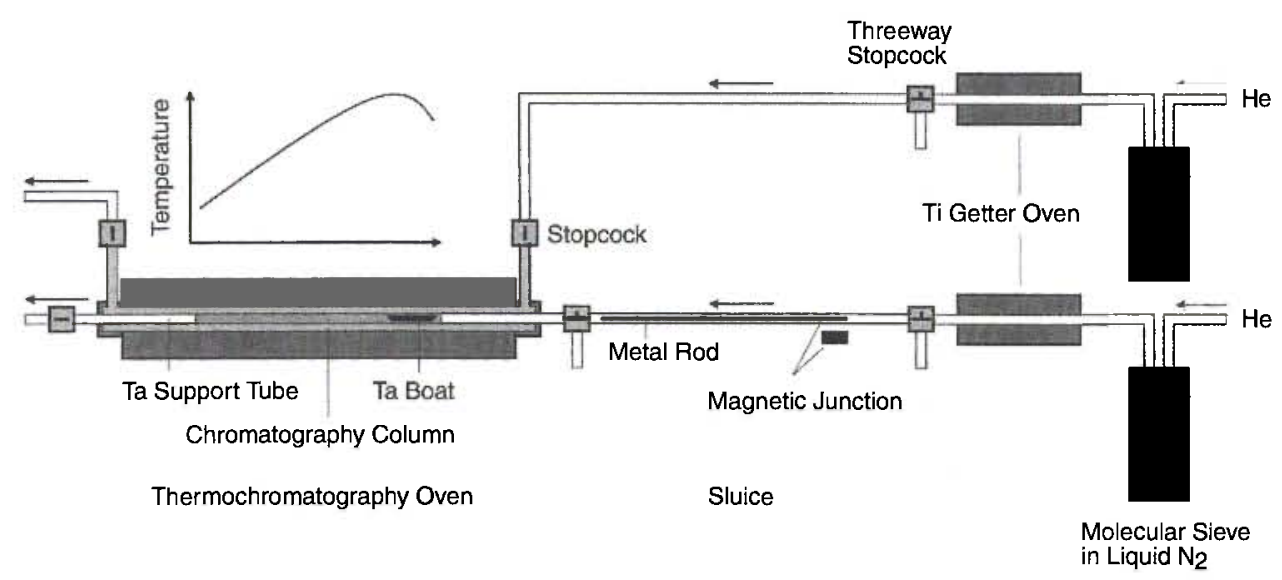

Fig. 1. The thermochromatographic apparatus.

The tube of the oven, made from alumina, is lined from inside with tantalum foil which is a very reactive getter material that adsorbes any oxygen emitted from the oven tube at working temperatures. The chromatographic columns are rolled from metal foils with impurity concentrations below $0.1 \%$. They were inserted into a support tube, also made from tantalum, which is mounted to both ends of the oven with Swagelok ${ }^{\oplus}$ tube fittings to be leak-proof.

Both the chromatography carrier gas and the gas for flushing the entire oven volume outside the support tube is helium with a purity of $99.999 \%$. The main impurities, $\mathrm{H}_{2} \mathrm{O}, \mathrm{CO}_{2}$ and $\mathrm{O}_{2}$, are removed with a molecular sieve at liquid nitrogen temperature and a titanium getter at $1250 \mathrm{~K}$. All gas pipes are made from stainless steel connected with Swagelok ${ }^{\circledast}$ fittings.

The sample is inserted into the apparatus through a sluice. It consists of a quartz tube between two threeway stopcocks. In the quartz tube there is a magnetic metal rod. With a strong external magnet at the outside of the quartz tube this rod can be moved in axial direction.

An experiment is started by preconditioning the apparatus at working temperature for about one hour. Then both sluice stopcocks are set to waste-gas position and a small tantalum boat with the sample is inserted into the quartz tube between oven and metal rod. After flusing the sluice with purified helium for at least 5 minutes, the sample boat is pushed using the metal rod into the thermochromatographic starting position, in the hottest zone of the oven.

After 30 minutes, the gradient oven heating is switched off. This terminates the chromatographic process, since the temperature falls about $100 \mathrm{~K}$ within a few seconds. The nuclide distribution on the column is determined off-line. After cutting the column in $1 \mathrm{~cm}$ pieces, their $\alpha$ or $\gamma$ activities are measured with PIPS or HPGe detectors, respectively.

\subsection{Actinide experiments}

The actinides under study were obtained by different ways. Initial experiments were carried out at the GSI
UNILAC accelerator with the ${ }^{248} \mathrm{Cm}+{ }^{22} \mathrm{Ne}$ reaction [8] using the actinide fractions of both ARCA and OLGA experiments [9]:

In the ARCA experiments the actinides were adsorbed onto cation exchange columns. The actinides were eluted from these columns with $6 \mathrm{M} \mathrm{HNO}_{3}$. The eluent was then evaporated to dryness and the resulting residue taken up in $1 \mathrm{M} \mathrm{NH}_{4} \mathrm{NO}_{3}$. The actinides were deposited electrolytically on a tantalum sheet from that solution. Finally, the activity was dissolved in $1 \mathrm{M} \mathrm{HNO}_{3}$.

In the OLGA experiments, the actinides are adsorbed in the first part of the quartz column and in the quartz wool plug. They were eluted with hot conc. $\mathrm{HNO}_{3}$. No further purification was carried out.

For both the ARCA and OLGA experiments, the last step was the evaporation of the final nitric acid solution in the tantalum sample boat. A small piece of La was added in order to reduce the actinides to the metallic state.

In further experiments, we used the ${ }^{248} \mathrm{Cm}+{ }^{18} \mathrm{O}$ reaction [10] both at the U-400 cyclotron of the Flerov Laboratory of Nuclear Reactions, Dubna, and at the Philips cyclotron at PSI. In both cases, the recoil nuclei of the nuclear reaction were caught in $5 \mu \mathrm{m} \mathrm{Ti}$ foils. These catcher foils could be used without further preparation as thermochromatographic samples.

Table 1 lists the experimental parameters of the thermochromatographic experiments.

\subsection{Data analysis}

To check the validity of the spectra obtained and to avoid numerical errors sometimes caused by automatic peak searching and fitting software, all alpha spectra were analyzed manually with the commercially available Microcal Origin Peak Fitting Module, which was customized for alpha spectroscopy. Half-life corrections were carried out with the calculated peak areas.

The adsorption temperature is derived from the adsorption position on the column. This temperature is connected with the adsorption enthalpy by the transport equation for ideal linear gas chromatography [11], 
Table 1. Experimental parameters

\begin{tabular}{ccccc}
\hline $\begin{array}{c}\text { Col- } \\
\text { umn }\end{array}$ & $\begin{array}{c}\text { Start } \\
\text { temper- } \\
\text { ature } \\
{[\mathrm{K}]}\end{array}$ & $\begin{array}{c}\text { Temperature } \\
\text { gradient } \\
{[\mathrm{K} / \mathrm{cm}]}\end{array}$ & $\begin{array}{c}\text { Normed col- } \\
\text { umn area } \\
{\left[\mathrm{cm}^{2} \text { per cm }\right.} \\
\text { column length }]\end{array}$ & $\begin{array}{c}\text { Carrier } \\
\text { gas flow } \\
{\left[\mathrm{cm}^{3} / \mathrm{min}\right]}\end{array}$ \\
\hline $\mathrm{Ti}$ & 1480 & 42.4 & 1.35 & 230 \\
$\mathrm{~V}$ & 1645 & 39.1 & 1.57 & 230 \\
$\mathrm{~V}$ & 1480 & 24.4 & 1.35 & 230 \\
$\mathrm{Nb}$ & 1645 & 39.1 & 1.57 & 230 \\
$\mathrm{Nb}$ & 1480 & 42.4 & 1.35 & 230 \\
$\mathrm{Nb}$ & 1480 & 24.4 & 1.35 & 230 \\
$\mathrm{Ta}$ & 1645 & 39.1 & 1.57 & 230 \\
$\mathrm{Ta}$ & 1480 & 42.4 & 1.35 & 230 \\
$\mathrm{Mo}$ & 1645 & 39.1 & 1.57 & 230 \\
$\mathrm{Mo}$ & 1480 & 24.4 & 1.35 & 230 \\
\hline
\end{tabular}

$$
\frac{\mathrm{d} x}{\mathrm{~d} t}=\frac{u(T)}{1+k_{i}}
$$

( $x$ - length coordinate of the column, $t-$ time, $u-$ linear gas velocity, $T$ - temperature, $k_{i}$ - corrected partition coefficient of the species $i$ ),

where $k_{i}$ is the corrected partition coefficient of the species $i$. This coefficient is a function of the adsorption enthalpy [12]:

$$
k_{i}=\frac{a \cdot V}{v \cdot A} \cdot \exp \left(\frac{-\Delta H_{\mathrm{ads}}^{\mathrm{o}}}{R T}\right) \cdot \exp \left(\frac{-\Delta S_{\mathrm{ads}}^{\mathrm{o}}}{R}\right)
$$

$(a-$ normalized column area, $v$ - normalized column volume, $V$ - molar standard volume, $A$ - molar standard area, $\Delta H_{\text {ads }}^{0}$ - standard adsorption enthalpy, $\Delta S_{\text {ads }}^{0}$ - standard adsorption entropy, $R$ - gas constant).

The algorithm from [13], implemented in the computer program TECRAD, was used to calculate with these equations the adsorption enthalpy from the adsorption temperature and the experimental parameters listed in Table 1.

\section{Results and discussion}

\subsection{Test of the apparatus with $\mathrm{Yb}$}

$\mathrm{Yb}$ was used for a test of the thermochromatographic apparatus, because this element is similar to the metallic divalent heavy actinides. ${ }^{169} \mathrm{Yb}$ was produced at the Rossendorf U-120 cyclotron in the ${ }^{166,167} \operatorname{Er}(\alpha, \mathrm{xn})$ reaction by irradiating a natural $\mathrm{Er}_{2} \mathrm{O}_{3}$-Target with $27 \mathrm{MeV}$ $\alpha$ particles. The ${ }^{169} \mathrm{Yb}$ was separated from the $\mathrm{Er}$ target with a standard $\alpha$-HIB procedure. The further preparation was similar to the preparation of the ARCA actinide fractions, as described in section 2.2.

In contrast to [5,6], and as already mentioned in the introduction, no $\mathrm{Ca}$ was used as reducing agent in the carrier gas, because this would modify the column surface and consequently make the interpretation of the results difficult.
V819: Mo Foll

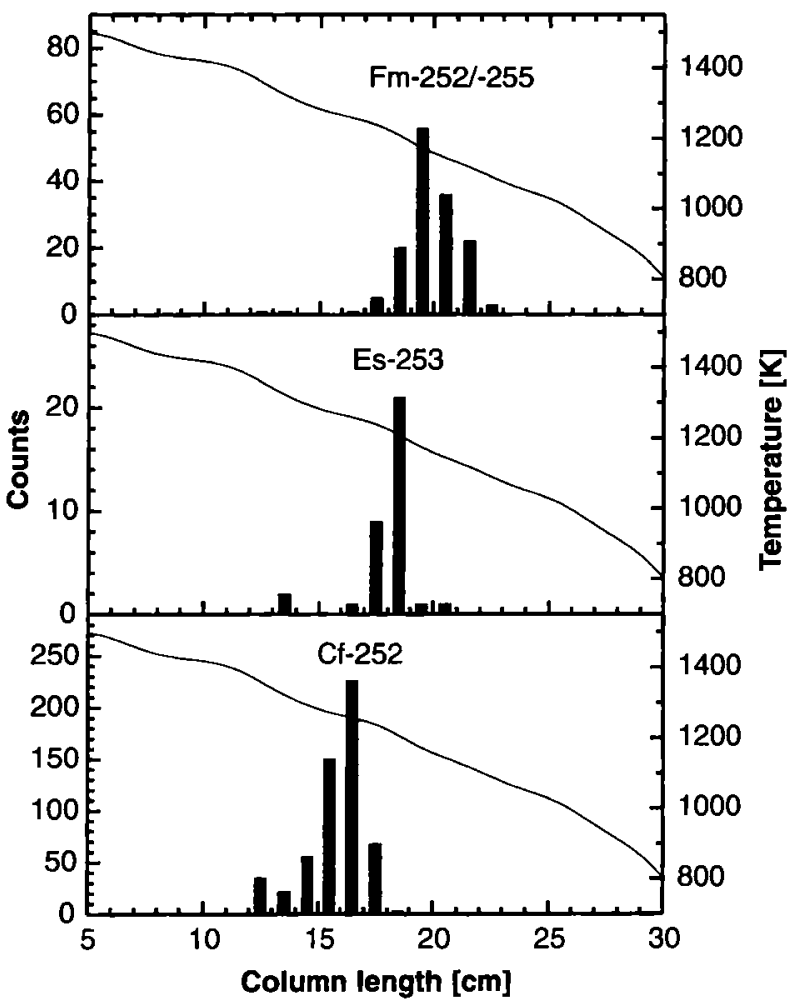

Fig. 2. Thermochromatogram of Cf, Es, and Fm on a Mo column; the activities were measured $3 \mathrm{~h}$ for Es and $\mathrm{Fm}$, and $20 \mathrm{~h}$ for $\mathrm{Cf}$.

On $\mathrm{Ti}$, the adsorption enthalpy is $-210 \mathrm{~kJ} / \mathrm{mol}$. This corresponds very well with previous experiments [5]. In a second experiment, we used a $\mathrm{Nb}$ column to evaluate the high temperature capabilities of our apparatus. The $\mathrm{Yb}$ adsorption enthalpy of $-326 \mathrm{~kJ} / \mathrm{mol}$ is also in good agreement with earlier results [7].

The high enthalpy values obtained in both experiments show unambiguously that $\mathrm{Yb}$ is adsorbed in the metallic state, without adding $\mathrm{Ca}$ as a reducing agent to the carrier gas. If oxygen were present the $\mathrm{Yb}$ would be transformed in an oxidized species and would have a much lower adsorption enthalpy [14].

\subsection{Adsorption measurements of the actinides}

Fig. 2 shows a typical nuclide distribution on a chromatographic column. The results are presented in different diagrams only for clarity, all species investigated in one thermochromatographic experiment are deposited simultaneously, of course.

The number of the actinide atoms used in our experiments were about $10^{3}, 10^{4}$, and $10^{6}$ atoms $\mathrm{Fm}$ 252/-255, Es-253, and Cf-252, respectively. Therefore, they do not influence each other in their adsorption behaviour.

In all experiments, without exception, a separation of $\mathrm{Cf}$, Es and Fm on the chromatographic column was 
Table 2. Adsorption enthalpies of this work together with previous data and EICHLER-MIEDEMA calculations; for Cf the metallic valency used in the calculations is also listed; former data of experiments using reducing metal vapours in the carrier gas are not reported

\begin{tabular}{|c|c|c|c|c|}
\hline \multirow[t]{2}{*}{ Actinide } & \multirow[t]{2}{*}{ Column } & \multicolumn{3}{|c|}{ Adsorption enthalpy $[\mathrm{kJ} / \mathrm{mol}]$} \\
\hline & & This work & Older data & Calculation \\
\hline $\mathrm{Cf}$ & $\begin{array}{c}\mathrm{Ti} \\
\mathrm{V} \\
\mathrm{Nb} \\
\mathrm{Ta} \\
\mathrm{Mo}\end{array}$ & $\begin{array}{c}-201 \\
-296,-302 \\
-290,-288 \\
-309 \\
-326,-305\end{array}$ & & $\begin{array}{l}-283 \text { (divalent) } \\
-207 \text { (trivalent) } \\
-340 \text { (divalent) } \\
-350 \text { (divalent) } \\
-360 \text { (divalent) } \\
-404 \text { (divalent) }\end{array}$ \\
\hline Es & $\begin{array}{c}\mathrm{Ti} \\
\mathrm{V} \\
\mathrm{Nb} \\
\mathrm{Ta} \\
\mathrm{Mo}\end{array}$ & $\begin{array}{c}-189 \\
-284,-276 \\
-268,-269 \\
-284 \\
-314,-291\end{array}$ & $\begin{array}{l}-220[7] \\
-290[7]\end{array}$ & $\begin{array}{l}-215 \\
-274 \\
-283 \\
-294 \\
-341\end{array}$ \\
\hline Fm & $\begin{array}{c}\mathrm{Ti} \\
\mathrm{V} \\
\mathrm{Nb} \\
\mathrm{Ta} \\
\mathrm{Mo}\end{array}$ & $\begin{array}{c}-182 \\
-271,-262 \\
-241,-250 \\
-267 \\
-284,-279\end{array}$ & $\begin{array}{l}-210[7] \\
-270[7] \\
-280[7] \\
-290[7]\end{array}$ & $\begin{array}{l}-210 \\
-263 \\
-266 \\
-275 \\
-314\end{array}$ \\
\hline
\end{tabular}

observed: Cf is always adsorbed at the highest, Fm always at the lowest temperature. In other words, the differences between the adsorption enthalpies of $\mathrm{Cf}$, $\mathrm{Es}$, and Fm are large enough to be detected with our method. The magnitude of the metallic properties of Es determining the adsorption behaviour must be intermediate to those of Cf and Fm.

Based on the MIEDEMA method for calculating alloy formation enthalpies [15, 16], EICHLER developed a model for understanding the adsorption enthalpy at zero coverage, such as those obtained in thermochromatographic experiments [17]. The basic idea is that the adsorption enthalpy is the sum of two terms. One term is the net adsorption heat, which can be deduced from the heat of solution of the adsorbed species in the solid adsorbens metal and the displacement energy. The second term is the sublimation enthalpy of the adsorbed substance. Calculations with this model show a rather good agreement with experimentally obtained adsorption enthalpy values. For most adsorbate systems, they predict the position of Es between $\mathrm{Cf}$ and Fm, like observed in our experiments. Table 2 lists our experimental adsorption enthalpies together with those calculated with the EICHLER-MIEDEMA model.

In addition to this model calculations, it is an experimentally well established fact that the adsorption heats of chemical similar substances correlates to their sublimation enthalpies, especially in case of metallic adsorbate bonding [18]. Furthermore, measurements of adsorption enthalpies of the lanthanides on $W$ and $R e$ showed, that the adsorption and desublimation enthalpies for the lanthanide series are not only proportional to one another but exhibit merely a parallel shift [19]. Consequently, because the metallic heavy actinides behave similarly to the lanthanides, one has to expect that the sublimation enthalpy of Es is between those of $\mathrm{Cf}$ and $\mathrm{Fm}$.

This stands in contrast to values obtained by direct vapour pressure measurements over $\mathrm{Yb}$ and $\mathrm{Sm}$ alloys of Es and Fm, $133 \mathrm{~kJ} / \mathrm{mol}$ [1] and $143 \mathrm{~kJ} / \mathrm{mol} \mathrm{[20],}$ resp., which show an opposite trend. Therefore, either the sublimation enthalpy reported for Es or for Fm should be corrected. We checked these values based on the correlation to the adsorption enthalpies.

For our calculations we choosed $\mathrm{Cf}$ as an internal standard, as mentioned in the introduction, because the sublimation behaviour of this metal is well-investigated [21]. Cf can exist both in the metallic tri- [21] and the divalent [2] state. Disregarding $\mathrm{Ti}$, comparing the magnitude of the adsorption enthalpies on all metals investigated with the EICHLER-MIEDEMA calculations indicate that $\mathrm{Cf}$ is adsorbed in the divalent state. (The deposition of $\mathrm{Cf}$ on $\mathrm{Ti}$, as part of our No experiments carried out recently, will be discussed in detail in [22].) Previous experiments on Mo columns indicated an adsorption of $\mathrm{Cf}$ in the trivalent state, due to the strong interaction with Mo [6]. This could not be reproduced in our experiments, which is further evidence for the $\mathrm{Cf}$ character of being borderline between tri- and divalency, depending on the preparation conditions, as discussed in [2]. Even if Cf is adsorbed in the trivalent state, however, one can expect the validity of a linear correlation between adsorption and sublimation heats as mentioned above, due to the smooth transition of the tri- to divalent character in the actinide series.

Based on this proportionality one can calculate the sublimation enthalpies of Fm and Es from the measured adsorption heats. Fig. 3 shows the procedure.

If the sublimation enthalpies of $\mathrm{Cf}$, Es, and Fm are correct, the adsorption sublimation enthalpy pairs 


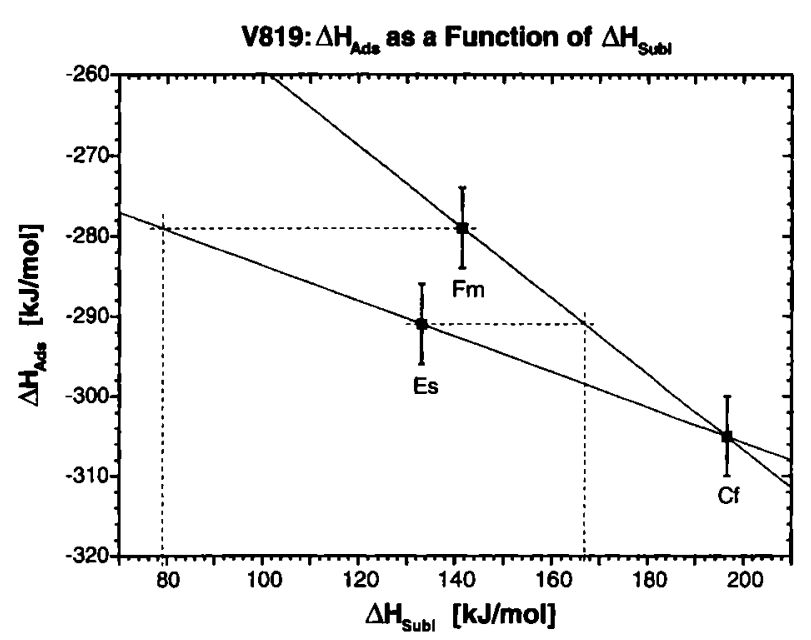

Fig. 3. Calculation of sublimation enthalpies of Es and Fm, based on the proportionality between adsorption and sublimation enthalpies.

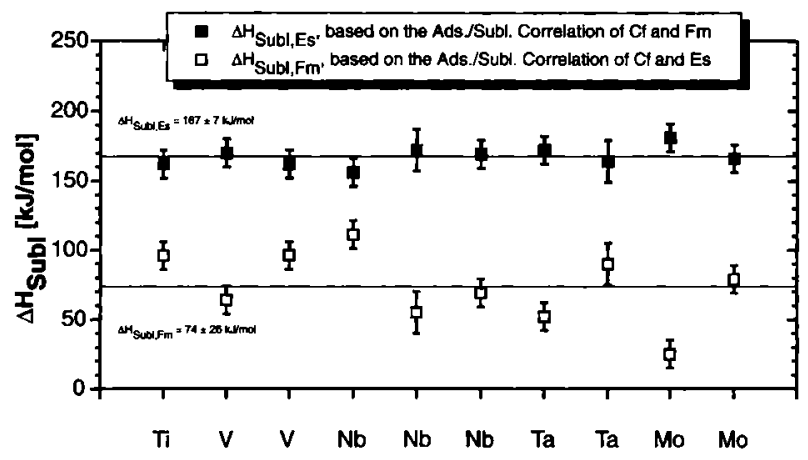

Fig. 4. Calculated sublimation enthalpies of Es and Fm.

should yield a straight line in the diagram. Because of the opposite trends in the adsorption and sublimation enthalpies mentioned above, this is obviously not the case. Because it has been investigated several times, one may assume that the Cf sublimation enthalpy is correct and there exist two possibilities: Either the Es sublimation enthalpy reported is correct and the $\mathrm{Fm}$ value is lower than that of Es (Fig. 3: about $80 \mathrm{~kJ} /$ mol), or the Fm value is correct then the Es sublimation enthalpy in Fig. 3 is about $167 \mathrm{~kJ} / \mathrm{mol}$.

The results of these calculations for all experiments are shown in Fig. 4. Assuming that the literature sublimation enthalpy of $\mathrm{Fm}(143 \mathrm{~kJ} / \mathrm{mol})$ is correct a sublimation heat for Es of $167 \pm 15_{95 \%} \mathrm{~kJ} / \mathrm{mol}$ results. If the literature value of Es is correct $(133 \mathrm{~kJ} / \mathrm{mol})$, the corresponding sublimation enthalpy of $\mathrm{Fm}$ would be $74 \pm 52_{95 \%} \mathrm{~kJ} / \mathrm{mol}$.

While the value for Es obtained is typical for a metallic divalent metal [2], the calculated sublimation enthalpy of Fm is far too small. Consequently, we consider the sublimation enthalpy of $\mathrm{Fm}$ reported in the literature to be more correct than that reported for Es. The latter is obviously too small in comparison to the Fm value.

The sublimation enthalpy of Es calculated here agrees well with the expected value, based on several

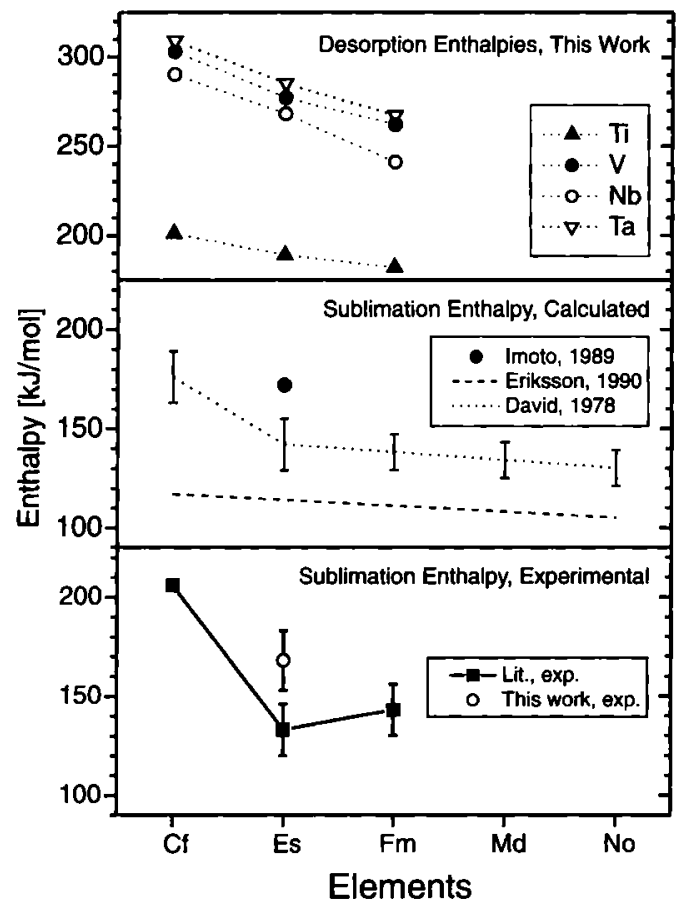

Fig. 5. Thermochromatographic desorption and macroscopic sublimation enthalpies of Cf, Es, and Fm.

theoretical calculations using different approaches $[23,24,25]$.

This indicates in agreement with theory [23], that the metallic divalency in the second half of the actinide series is fully developed only at Fm and beyond. This is also supported by experiments with No carried out recently [22].

The agreement of our results with theory is shown graphically in Fig. 5, depicting the adsorption data of this work together with theoretically and experimentally obtained sublimation enthalpies of Cf, Es and Fm. The similar trends of the adsorption and sublimation enthalpies emphasize the possibility to derive sublimation enthalpies from adsorption experiments.

\section{Acknowledgments}

The authors gratefully acknowledge the continuous interest of Prof. $H$. Nitsche. This work was supported by both DFG, contract Hu642/1-1 and BMBF, contract 06DR666I(4)/1.

\section{References}

1. Kleinschmidt, P. D., Ward, J. W., Matlack, G. M., Haire, R. G.: The Cohesive Energy of Einsteinium Metal, High Temp. Sci. 19, 267 (1985).

2. Johansson, B., Brooks, M. S. S.: "Theory of Cohesion in Rare Earths and Actinides", Handbook on the Physics and Chemistry of Rare Earths, Vol. 17 - Lanthanides/Actinides: Physics - I, p. 149, Gschneidner, K. A., Jr., Eyring, L., Lander, G. H., Choppin, G. R. (Eds.), Elsevier 1993.

3. Morss, L.: "Comparative Thermochemical and OxidationReduction Properties of Lanthanides and Actinides", Handbook on the Physics and Chemistry of Rare Earths, 
Vol. 18 - Lanthanides/Actinides: Chemistry - I, p. 239, Gschneidner, K. A., Jr., Eyring, G. H., Choppin, L., Lander, G. R. (Eds.), Elsevier 1994.

4. Keller, O. L., Jr.: "Chemistry of the Heavy Actinides and Light Transactinides", Radiochim. Acta 37, 169 (1984).

5. Hübener, S., Zvara, I.: "The Thermochromatography of Metallic Elements in Titanium Columns", Radiochim. Acta 27, 157 (1980).

6. Hübener, S., Zvara, I.: "Characterization of some Metallic State Properties of Mendelevium and other Actinoides by Thermochromatography", Radiochim. Acta 31, 89 (1982).

7. Hübener, S., Eichler, B., Schädel, M., Brüchle, W., Gregorich, K. E., Hoffman, D. C.: "Thermochromatographic studies of heavy actinoides in metal columns", J. Alloys Comp. 213/214, 429 (1994).

8. Lee, D., v. Gunten, H. R., Jacak, B., Nurmia, M., Yuan-fang Liu, Cheng Luo, Seaborg, G. T., Hoffman, D. C.: "Production of heavy actinides from interactions of ${ }^{16} \mathrm{O},{ }^{18} \mathrm{O}$, ${ }^{20} \mathrm{Ne}$, and ${ }^{22} \mathrm{Ne}$ with ${ }^{248} \mathrm{Cm}$ ", Phys. Rev. C 25, 286 (1982).

9. Schädel, M., et al.: "Chemistry with Element 106, Seaborgium - The Isotopes ${ }^{265} \mathrm{Sg}$ and ${ }^{266} \mathrm{Sg}$ ", GSI Scientific Report 1995 GSI 96-1, 10 (1996).

10. Lee, D., Moddy, K. J., Nurmia, M. J., Seaborg, G. T., v. Gunten, H. R., Hoffman, D. C.: "Excitation functions for production of heavy actinides from interactions of ${ }^{18} \mathrm{O}$ with ${ }^{248} \mathrm{Cm}$ and ${ }^{249} \mathrm{Cf}$ ", Phys. Rev. C 27, 2656 (1983).

11. Leibnitz, E., Struppe, H.-G.: Handbuch der Gaschromatographie, Akademische Verlagsgesellschaft Geest \& Portig, Leipzig 1984, p. 50.

12. Eichler, B., Zvara, I.: "Evaluation of the Enthalpy of Adsorption from Thermochromatographic Data", Radiochim. Acta 30, 233 (1982).

13. Funke, H., Hübener, S., Roß, A., Eichler, B.: "Contributions to the Evaluation of Thermochromatographic Experiments", FZR, Institute of Radiochemistry, Annual Report 1993, FZR-43, 53 (1994).

14. Eichler, B., Hübener, S., Roßbach, H.: "Adsorption flüchtiger Metalle auf metallischen Oberflächen und ihre Anwen- dung in der Kernchemie - Berechnung der Enthalpie der dissoziativen Chemisorption von Monoxiden der Seltenerdmetalle und Actinoide", ZfK-Report, ZfK-609 (1986).

15. Miedema, A. R., Boom, R., de Boer, F. R.: "On the Heat of Formation of Solid Alloys", J. Less-Common Met. 41, 284 (1975).

16. De Boer, F. R., Boom, R., Mattens, W. C. M., Miedema, A. R., Niessen, A. K.: "Cohesion in Metalls: Transition Metal Alloys", Cohesion and Structure, Vol. 1, de Boer, F. R., Pettifor, D. G. (Eds.), North-Holland 1988.

17. Eichler, B.: "Bestimmung der Adsorptionswärme gasförmiger Metalle auf festen Metalloberflächen (Empirisches Modell)", ZfK-396 (1979).

18. Eichler, B.: "Trennung von Radionukliden durch Verflüchtigungsprozesse", Thesis, Dresden University of Technology (1976).

19. Nikulin, V. K., Potechina, N. D.: "Effekt izmeneniya valentnovo sostoyaniya atomov redkosemelnych elementov pri adsorbcii na perevodnych metallach", Fiz. Tverd. Tela 20, 3354 (1978).

20. Haire, R. G., Gibson, J. K.: "The enthalpy of sublimation and thermodynamic functions of fermium", J. Chem. Phys. 91, 7085 (1989).

21. Ward, J. W.: "Electronic Structure and Bonding in Transuranics: Comparison with Lanthanides", J. Less-Common Met. 93, 279 (1983).

22. Taut, S., Hübener, S., et al.: "Thermochromatography of No on Metal Surfaces", to be published.

23. Eriksson, O., Brooks, M. S. S., Johansson, B.: "Calculated Cohesive Properties of Lanthanide and Lanthanide-Like Actinide Elements", J. Less-Common Met. 158, 207 (1990).

24. David, F., Samhoun, K., Guillaumont, R., Edelstein, N.: "Thermodynamic Properties of $5 f$ Elements", J. Inorg. Nucl. Chem. 40, 69 (1978).

25. Imoto, S.: "Computed Energy Levels of Actinide Configurations", J. Nucl. Mat. 166, 68 (1989). 